\title{
Coherent Rayleigh-Brillouin Scattering: Influence of the Intermolecular Potential
}

\author{
Lei $\mathrm{Wu}^{*}$, Craig White*, Thomas J. Scanlon*, Jason M. Reese ${ }^{\dagger}$ and Yonghao Zhang* \\ *James Weir Fluids Laboratory, Department of Mechanical and Aerospace Engineering, University of \\ Strathclyde, Glasgow G1 1XJ, UK \\ ${ }^{\dagger}$ School of Engineering, University of Edinburgh, Edinburgh EH9 3JL, UK
}

\begin{abstract}
The spectrum of the coherent Rayleigh-Brillouin scattering (CRBS) of light by a rarefied gas is obtained by solving the Boltzmann equation numerically using the fast spectral method. The influence of the intermolecular potential on the CRBS spectrum is investigated and the accuracy of the prevailing Tenti's s6 kinetic model is evaluated. Our numerical results show that i) the intermolecular potential has a great influence on CRBS spectrum when the Knudsen number is between 0.05 and 1 and ii) Tenti's s6 kinetic model can only predict the line shape accurately for Maxwell gases at small Knudsen numbers.
\end{abstract}

Keywords: Coherent Rayleigh-Brillouin scattering, Boltzmann equation, Fast spectral method

PACS: $51.10 .+\mathrm{y}, 51.20 .+\mathrm{d}, 47.11 . \mathrm{Kb}$

\section{INTRODUCTION}

Density fluctuations in gases, which can be measured by the spontaneous and coherent Rayleigh-Brillouin scattering (RBS), contain information such as the temperature and bulk viscosity. This information can be extracted by comparing the experimental and theoretical spectra, where the accuracy of the obtained information depends on how reliable the experimental and theoretical results are. In the past few years, rapid improvements in the experimental resolution have been achieved [1, 2, 3], however, accurate theoretical line shapes (spectra) are lacking, although several kinetic models have been proposed $[4,5,6,7]$.

The Knudsen number $K n$ (ratio between the molecular mean free path and the scattering wavelength) is an important parameter determining the line shape. When $K n$ is small, the gas is in the hydrodynamic regime and the scattering process is described by the Navier-Stokes (NS) equations. On the other hand, when Kn approaches infinity, the gas is in the collisionless regime and the spectrum is Gaussian [8]. Accurate theoretical line shapes are difficult to obtain in the kinetic regime $0.05<K n<1$, as the intricate Boltzmann equation (BE) must be solved. However, this regime is of significant interest since the line shape is sensitive to gas states, which allows accurate measurement of gas properties including the bulk viscosity [2].

Dimensional analysis implies that the line shape also depends on the intermolecular potential. However, spontaneous RBS experiments and theory $[9,10,11]$ have shown that the intermolecular potentials do not affect the line shapes of monatomic gases in the kinetic regime. Whether this conclusion applies to coherent RBS (CRBS) or not has never been checked systematically, since CRBS line shapes are different to those of spontaneous RBS as density fluctuations are induced by the optical dipole force, instead of arising spontaneously in thermal equilibrium $[1,5]$.

Due to the complexity of the BE, Tenti's s6 kinetic model, which simplifies the intricate Boltzmann collision operator, is widely used and its line shapes are regarded as the most accurate $[4,6]$. In this paper, we investigate the influence of the intermolecular potential on the CRBS line shape and evaluate the accuracy of the s6 kinetic model using a deterministic numerical method, where we solve the Boltzmann collision operator using the fast spectral method $[12,13]$.

\section{LINEARIZED BOLTZMANN EQUATION}

In CRBS experiments, the molecules are under the optical confinement induced by the interference of two pump beams: the acceleration of an individual molecule is $a_{0} \cos \left[2 \pi\left(x / \lambda-f_{d} t\right)\right]$, where $\lambda$ is the effective wavelength, $f_{d}$ is the frequency difference between the two pump beams, and $a_{0}$ describes the strength of the optical potential. When the beam intensities are small, the spectrum of the scattered light can be obtained by solving the linearized BE; for 
monatomic gases, the velocity distribution function $f$ can be expressed by $f=f_{e q}+h$, where $f_{e q}(\mathbf{v})=\pi^{-3 / 2} e^{-|\mathbf{v}|^{2}}$ is the global equilibrium distribution function and $h$ is the perturbed velocity distribution function. The evolution of the perturbed distribution function is governed by

$$
\frac{\partial h}{\partial t}+v_{x} \frac{\partial h}{\partial x}-\cos \left(x-f_{d} t\right) v_{x} f_{e q}(\mathbf{v})=\mathscr{L}_{g}(h)-v_{e q}(\mathbf{v}) h,
$$

where

$$
\mathscr{L}_{g}(h)=\iint B\left[2 f_{e q}\left(\mathbf{v}^{\prime}\right) h\left(\mathbf{v}_{*}^{\prime}\right)-f_{e q}(\mathbf{v}) h\left(\mathbf{v}_{*}\right)\right] d \Omega d \mathbf{v}_{*},
$$

could be viewed as the gain part of the linearized Boltzmann collision term, and

$$
v_{e q}(\mathbf{v})=\iint B f_{e q}\left(\mathbf{v}_{*}\right) d \Omega d \mathbf{v}_{*}
$$

is the equilibrium collision frequency. Note that the molecular velocity $\mathbf{v}$, the spatial coordinate $x$, and the time have been respectively normalized by the most probable velocity $v_{m}=\sqrt{2 k_{B} T_{0} / m}$, the effective wavelength, and $\lambda / v_{m}$, with $k_{B}$ being the Boltzmann constant, $T_{0}$ the equilibrium temperature, and $m$ the molecular mass.

The Boltzmann collision kernel $B$ determines the gas transport properties. Here we consider the inverse power-law intermolecular interaction, where the shear viscosity $\mu$ is proportional to $T^{\omega}$, with $\omega$ being the viscosity index whose values for typical gases can be found in [14]; specifically, the hard-sphere and Maxwell gas has a viscosity index of 0.5 and 1 , respectively. We choose the collision kernel to be $[12,13]$

$$
B=5\left|v-v_{*}\right|^{2(\omega-1)} \sin ^{1 / 2-\omega} \theta / 2^{15 / 2-2 \omega} \Gamma^{2}\left(\frac{9-2 \omega}{4}\right) K n,
$$

where $\theta$ is the deflection angle after the binary collision, $\Gamma$ is the gamma function, and the Knudsen number is

$$
K n=\frac{\mu\left(T_{0}\right)}{n_{0} \lambda} \sqrt{\frac{\pi}{2 m k_{B} T_{0}}},
$$

with $n_{0}$ being the average molecular number density.

To find the CRBS spectrum, we take the Fourier transformation of Eq. (1) in the temporal and spatial domains, and solve the resultant equation in an iterative manner. That is, given the frequency difference $f_{d}$, Knudsen number $K n$, and viscosity index $\omega$, we have

$$
\tilde{h}^{j+1}(\mathbf{v})=\frac{v_{x} f_{e q}(\mathbf{v})+\mathscr{L}_{g}\left(\tilde{h}^{j}(\mathbf{v})\right)}{2 \pi i f_{d}-2 \pi i v_{x}+v_{e q}(\mathbf{v})},
$$

where $\tilde{h}$ is the spatial-temporal Fourier transformation of $h$ and $j$ denotes the iteration step. The linearized Boltzmann colliison operator $\mathscr{L}_{g}(\tilde{h})$ and the equilibrium collision frequency $v_{e q}$ can be solved by the fast spectral method [13, 15]. Starting from $\tilde{h}=0$, the iteration is terminated when the relative error of $\tilde{h}$ between two consecutive iteration steps is less than $10^{-8}$. The spectrum is then given by

$$
S\left(K n, \omega, f_{d}\right)=\left|\int h d \mathbf{v}\right|^{2},
$$

where the frequency difference $f_{d}$ is normalized by the characteristic frequency $\sqrt{2 k_{B} T_{0} / m} / \lambda$.

Since the fast spectral method solves the BE in velocity space efficiently and no spatial and temporal variables are involved, it takes about one minute (on a PC with an Intel Xenon $3.3 \mathrm{GHz} \mathrm{CPU}$, only one core is used) to get one line shape, while the computation time using the direct simulation Monte Carlo (DSMC) method is significant [16]. Roughly speaking, our method can be faster than the DSMC method by three orders of magnitude.

CRBS spectra for monatomic gases, obtained from the fast spectral method, are depicted in Figs. 1 and 2. Due to symmetry, only the positive frequency difference is shown. We plot the spectra for the Maxwell $(\omega=1)$ and hard sphere gases $(\omega=0.5)$ and, for comparison, the spectra are normalized by their maximum values. For other inverse power-law potentials with $0.5<\omega<1$, the spectra lie between the spectra of the Maxwell and hard sphere gases. When $K n<0.03$, the gas is in the hydrodynamic regime and the CRBS spectra can be well described by the NS equations, and there is no difference between different molecular models, as shown Fig. 1. As Kn increases, the NS equations fail 


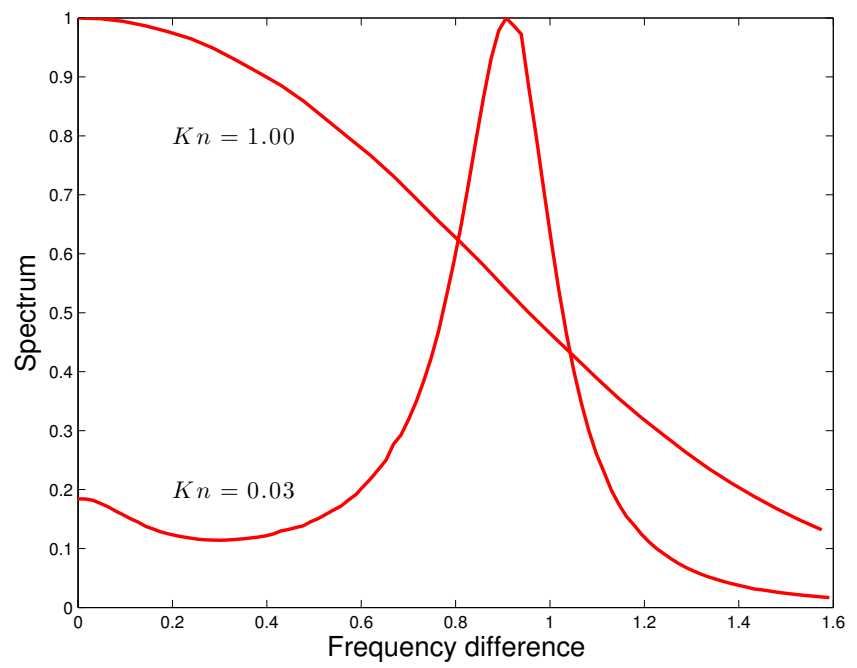

FIGURE 1. CRBS spectra at $K n=0.03$ and 1 . The difference between different potentials is indistinguishable.

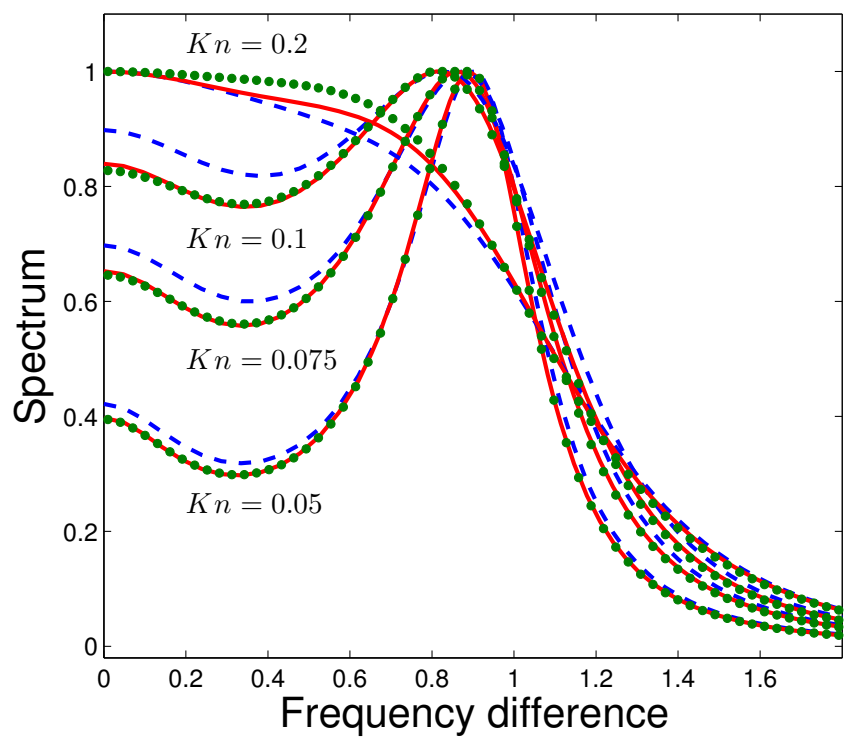

FIGURE 2. Comparisons of CRBS spectra between the hard sphere (dashed lines) and Maxwell (solid lines) monatomic gases. The dots are the results from the s6 kinetic model derived for Maxwell molecules [6].

to describe the rarefaction effect and differences between molecular models appear. The relative difference in spectra between the Maxwell and hard sphere gases is up to about $10 \%$ at zero frequency difference, when $K n \sim 0.1$, see Fig. 2. When $K n>1$, however, the spectrum is Gaussian and there is again no difference between molecular models, see Fig. 1.

We have also compared the CRBS spectra with those obtained from Tenti's s6 model [4]. From Fig. 2 we see that, for Maxwell molecules, the differences between our numerical results and those from Tenti's model are negligible when $K n \leq 0.1$. This is due to the fact that the collision frequency in both Tenti's kinetic model and the linearized Boltzmann equation is independent on the molecular velocity. The difference between our numerical results and those from Tenti's model grows when $K n>0.1$ and vanishes when $K n>1$. For hard-sphere molecules, the difference between our numerical results and those from Tenti's model is highly significant when $K n>0.05$ and vanishes when 


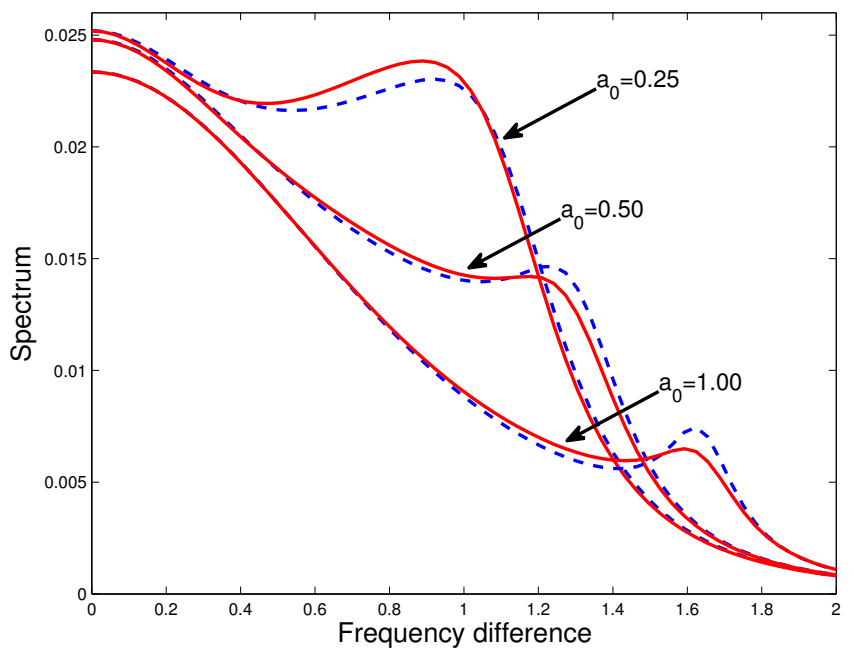

FIGURE 3. Comparisons of CRBS spectra between the hard sphere (dashed lines) and Maxwell (solid lines) monatomic gases, when the optical potential is high. The Knudsen number of the initial equilibrium state is $K n=0.1$.

$K n>1$.

\section{FULL BOLTZMANN EQUATION}

When the intensity of the two pump beams becomes appreciable (but has not yet reached the ionization threshold), the full Boltzmann equation should be considered:

$$
\frac{\partial f}{\partial t}+v_{x} \frac{\partial f}{\partial x}-a_{0} \cos \left(x-f_{d} t\right) \frac{\partial f}{\partial v_{x}}=Q\left(f, f_{*}\right)
$$

where $Q$ is the Boltzmann collision operator. We refer to [12] for the detailed form of the Boltzmann collision operator and its fast spectral approximation.

Figure 3 shows the CRBS spectra at different values of optical strength $a_{0}$, where, initially, the gas is in global equilibrium. Obviously, the molecular potential affects the CRBS spectrum, as in the linear case. The comparison between Figs. 2 and 3 shows that the CRBS spectra in the linear and nonlinear regimes are quite different. Firstly, as $a_{0}$ increases, the Rayleigh scattering becomes increasingly important. Secondly, as $a_{0}$ increases, the position of the Brillouin peak shifts toward larger frequency difference, probably due to the larger temperature increase. Finally, the full width half maximum of the CRBS spectrum decreases as $a_{0}$ increases.

\section{CONCLUSIONS}

We have solved both the linear and nonlinear Boltzmann equation numerically using the fast spectral method. The deterministic numerical solver we have used is much faster than the DSMC method. We have found that the intermolecular potential greatly affects the CRBS line shapes. Although we only consider monatomic gases, we believe that the intermolecular potential also affects the CRBS line shapes of polyatomic gases. Our future work is to propose a kinetic equation to model polyatomic gases.

\section{REFERENCES}

1. M. O. Vieitez, E. J. van Duijn, W. Ubachs, B. Witschas, A. Meijer, A. S. de Wijn, N. J. Dam, and W. van de Water, Phys. Rev. A 82, 043836 (2010). 
2. Z. Gu, and W. Ubachs, Opt. Lett. 38, 1110 (2013).

3. A. Gerakis, M. N. Shneider, and P. F. Barker, Opt. Letts. 38, 4449 (2013).

4. G. Tenti, C. Boley, and R. Desai, Can J. Phys. 52, 285 (1974).

5. X. Pan, M. N. Shneider, and R. B. Miles, Phys. Rev. Lett. 89, 183001 (2002).

6. X. Pan, M. N. Shneider, and R. B. Miles, Phys. Rev. A 69, 033814 (2004).

7. W. Marques, J. Stat. Phys. p. 03013 (2007).

8. J. H. Grinstead, and P. F. Barker, Phys. Rev. Lett. 85, 1222 (2000).

9. A. Sugawara, S. Yip, and L. Sirovich, Phys. Fluids 11, 925 (1968).

10. N. A. Clark, Phys. Rev. A 12, 232 (1975)

11. V. Ghaem-Maghami, and A. D. May, Phys. Rev. A 22, 692 (1980).

12. L. Wu, C. White, T. J. Scanlon, J. M. Reese, and Y. H. Zhang, J. Comput. Phys. 250, 27-52 (2013).

13. L. Wu, J. M. Reese, and Y. H. Zhang, J. Fluid Mech. 746, 53-84 (2014).

14. S. Chapman, and T. Cowling, The Mathematical Theory of Non-uniform Gases, Cambridge University Press, 1970.

15. L. Wu, J. M. Reese, and Y. H. Zhang, J. Fluid Mech. 748, 350-367 (2014).

16. B. M. Cornella, S. F. Gimelshein, M. N. Shneider, T. C. Lilly, and A. D. Ketsdever, Opt. Exp. 20, 12975 (2012). 